\title{
Pathological Laughter Prodromal to a Stroke of the Head of the Left Caudate Nucleus
}

\author{
Matthew Beth Urhoy ${ }^{a}$ William J. House ${ }^{b}$ \\ aDepartment of Medicine, UMass Medical School, Baystate Medical Center, Springfield, MA, \\ USA; ${ }^{b}$ Neurosciences Division, UMass Medical School, Baystate Medical Center, Springfield, \\ MA, USA
}

\section{Keywords}

Laughter $\cdot$ Caudate nucleus $\cdot$ Stroke $\cdot$ Headache $\cdot$ Rash $\cdot$ Atrial myxoma

\begin{abstract}
A 38-year-old right-handed female with a past history of intermittent painful rash, dizzy spells, and chronic daily headaches as well as episodic migraines experienced an episode of transient involuntary pathological laughter, right arm weakness, and expressive dysphasia. She was found on MRI to have multiple strokes in multiple vascular distributions, including one in the head of the left caudate. A cardiac ECHO found an atrial myxoma, with extensive evaluation for other causes of stroke unrevealing. The differential diagnosis for pathological laughter in this patient is discussed. The most plausible cause in this patient is an infarct to the head of the left caudate nucleus caused by an embolus of the atrial myxoma.
\end{abstract}

\section{Introduction}

Laughter is a complex emotion that involves coordination among many areas of the brain responsible for comprehension, subjective interpretation of stimuli, emotion, and control of the muscles used to respond. Fou rire prodromique is a rare phenomenon of involuntary laughter leading up to a stroke. It was first described by Féré [1]. As laughter is such a complex expression, multiple pathological causes occur, most commonly diffuse or multifocal diseases such as pseudobulbar palsy, and have included various types of lesions to 

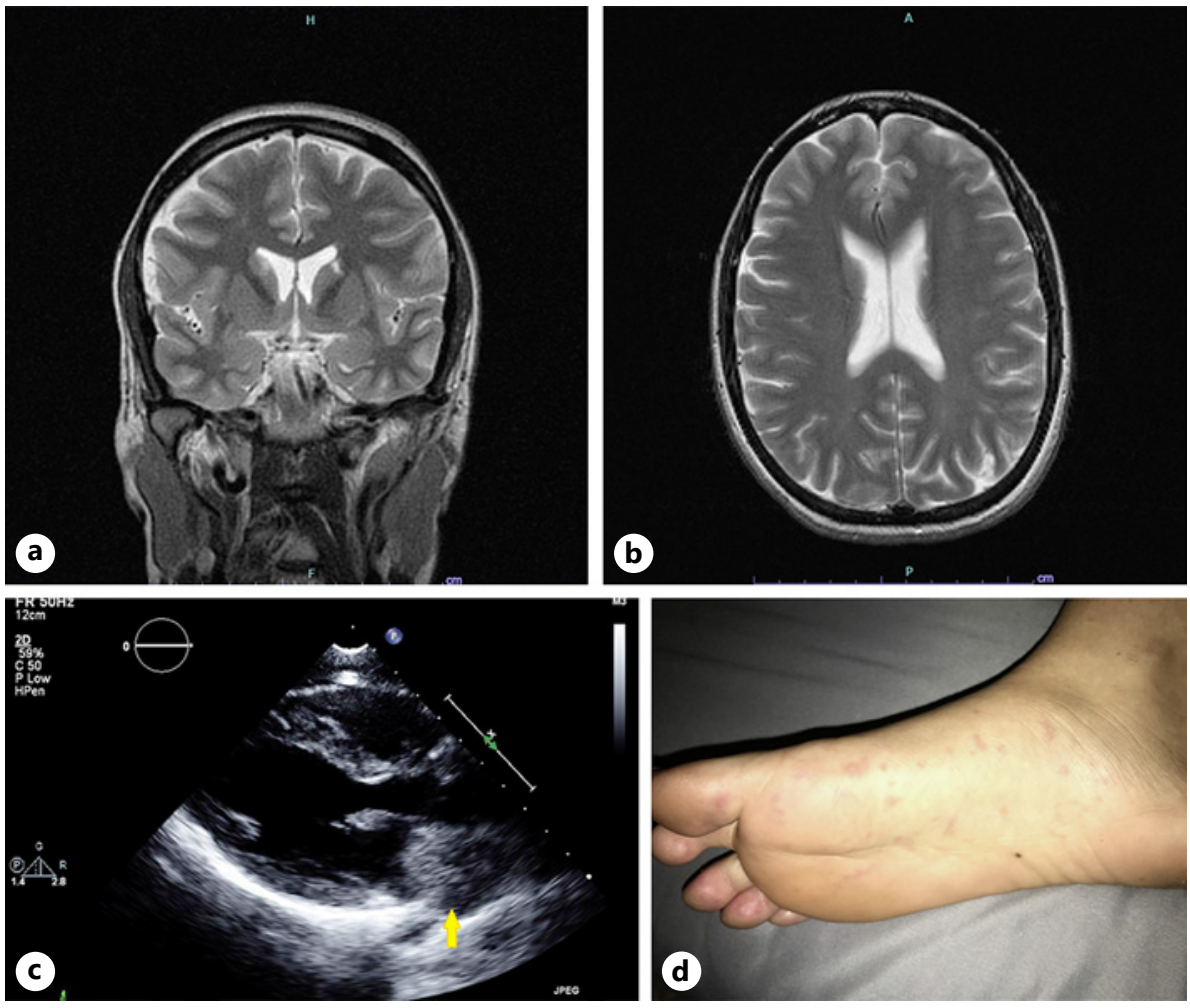

Fig. 1. Region of hyperintensity can be seen in the head of the left caudate nucleus on coronal T2 blade MRI (a) and on axial T2 MRI (b). Parasternal long axis view of the heart via transthoracic echocardiogram showing a left atrial mass approximately $3 \times 3 \mathrm{~cm}$ marked by the yellow arrow (c). Picture of the purpuric and tender macules taken by the patient at the time of their occurrence (d).

different areas of the brain [2-8]. We report a case of a patient who presented with pathological laughter prodromal to left arm weakness and expressive dysphasia due to an unusual cause.

\section{Case Report}

The patient was a 38-year-old right-handed female who began involuntarily laughing while having intercourse with her boyfriend. Her boyfriend reported that she was laughing uncontrollably for 5 minutes and moving around "like a fish out of water," and then she lost consciousness. When she regained consciousness, she realized she could not move her right arm.

She was then extremely fatigued and slept. Upon awakening, she realized she had word finding difficulties that lasted a week. She could not coordinate movement of her weak right arm for 2 days, and then control returned but the weakness lasted another 2 weeks.

She reported a past medical history of intermittent rashes on her extremities (shown in Fig. 1d), at times painful, sometimes following strenuous physical activity. An extensive evaluation for the cause of the rash had been done, including testing for autoimmune and infectious diseases as well as deficiency states, and were unrevealing.

She also reported that since childhood, she had episodic migraine headaches with visual aura, occurring once or twice a month. For a few years, she also had chronic daily headaches 
and dizzy spells not clearly vertiginous, but during which she would sit down until they passed. As a result, 16 months prior to the event, a head MRI without gadolinium and repeat labs were done, both unrevealing.

She worked as an arborist. She did not smoke or use other drugs. She took analgesic medicines infrequently. A neurological consultation was done 2 weeks after the event; general and neurological examination was normal.

A second brain MRI without gadolinium was ordered. It showed multiple subacute and chronic strokes in multiple vascular distributions, some hemorrhagic, including a chronic lesion in the head of the left caudate nucleus (shown in Fig. 1a, b).

Further workup included an unrevealing CT angiogram of her head and neck and unrevealing labs including blood cultures. An echocardiogram revealed a $3 \times 3 \mathrm{~cm}$ highly mobile left atrial myxoma (shown in Fig. 1c). She underwent surgical removal of the myxoma.

Five months after her cardiac surgery, follow-up neurological evaluation continued to be normal. Her intermittent rash, chronic daily headaches, and dizzy spells have not recurred. She has finished cardiac rehabilitation and just started back to work as an arborist. She continues to get migraines with visual aura a couple of times a month, unchanged from her presurgical pattern.

\section{Discussion/Conclusion}

Although atrial myxomas are the most common primary cardiac tumor, their incidence is quite rare. Atrial myxomas commonly embolize [9] and cause downstream arterial occlusion, causing symptoms such as purpuric rashes and strokes [10].

Episodes of physical stress or physical exercise could cause an increase in cardiac output leading to fragmentation and embolization of the tumor, which could explain the onset of new purpuric and tender macules and strokes following such activity in our patient.

Given the unrevealing extensive evaluation for other causes, we believe that this patient's atrial myxoma embolized, causing transient distal blockages in her extremities, leading to the cutaneous lesions, and the lesion of the head of the left caudate nucleus causing the pathological laughter. The mechanism of the resolved chronic daily headaches and dizzy spells is uncertain. Migraine with aura has been reported to resolve with surgery for atrial myxomas [11] but to our knowledge, not chronic daily headaches.

Cases of pathological laughter have been attributed to localized strokes of many different areas of the brain $[3,5-8]$. Lesions to the head of the caudate nucleus as a cause of pathological laughter are quite rare but have been previously reported [2, 4]. We believe that this is the first report of fou rire prodromique caused by an atrial myxoma.

\section{Statement of Ethics}

The paper is exempt from ethics committee approval because it is a retrospective analysis of 1 clinical case under the standard of care and intended for educational purposes. Written informed consent was obtained from the patient to publish her case (including publication of images).

\section{Conflict of Interest Statement}

The authors have no conflicts of interest to declare.

\section{Karger'}


Beth Urhoy and House: An Atypical Case of Involuntary Pathological Laughter

\section{Funding Sources}

This manuscript did not receive any funding.

\section{Author Contributions}

Both authors M.B. and W.H. contributed to the writing of this case report and agree to be accountable for all aspects of the work in ensuring that questions related to the accuracy or integrity of any part of the work are appropriately investigated and resolved.

\section{Data Availability Statement}

Data sharing is not applicable to this study as no datasets were generated or analyzed. All data generated or analyzed during this study are included in this article.

\section{References}

1 Féré MC. Le fou rire prodromique. Rev Neurol. 1903;11:353-8.

2 Lee S, Kim DY, Kim JS, Manda S, Danilov L, El-Refai M, et al. When should brain imaging be performed?: a case report of caudate nucleus infarct. Psychiatry. 2010;7(1):31-3.

3 Wali GM. "Fou rire prodromique" heralding a brainstem stroke. J Neurol Neurosurg Psychiatry. 1993;56(2): 209-10.

4 Carel C, Albucher JF, Manelfe C, Guiraud-Chaumeil B, Chollet F. Fou rire prodromique heralding a left internal carotid artery occlusion. Stroke. 1997 Oct;28(10):2081-3.

5 Dabby R, Watemberg N, Lampl Y, Eilam A, Rapaport A, Sadeh M. Pathological laughter as a symptom of midbrain infarction. Behav Neurol. 2004;15(3-4):73-6.

6 Ceccaldi M, Milandre L. A transient fit of laughter as the inaugural symptom of capsular-thalamic infarction. Neurology. 1994;44(9):1762.

7 Dulamea AO, Matei C, Mindruta I, Ionescu V. Pathological laughter as prodromal manifestation of transient ischemic attacks: case report and brief review. BMC Neurol. 2015;15:196.

8 Elyas AE, Bulters DO, Sparrow OC. Pathological laughter and crying in patients with pontine lesions. J Neurosurg Pediatr. 2011 Dec;8(6):544-7.

9 Wen XY, Chen YM, Yu LL, Wang SR, Zheng HB, Chen ZB, et al. Neurological manifestations of atrial myxoma: a retrospective analysis. Oncol Lett. 2018;16(4):4635-9.

10 Lee HJ, Park JY, Kim YS, Shin DH, Choi JS, Kim KH, et al. Cardiac myxoma diagnosed by signs of purpuric macules on both palms and soles. Ann Dermatol. 2012;24(3):337-40.

11 De Cuester L, van Diepen T, Koehler PJ. Migraine with aura triggered by cardiac myxoma: case report and literature review. Cephalgia. 2010 Nov;30(11):1396-9. 